\title{
Políticas sociales en discapacidad: una aproximación desde las acciones del Estado en Argentina
}

\section{Social policies related to disabilities: an approach from the actions of the State in Argentina}

\section{Resumen}

En Argentina, el I 3 \% de la población tiene algún tipo de discapacidad, condición que implica desigualdades en la participación social de este conjunto. El marco normativo vigente resulta avanzado en la materia aunque no llega a generar acciones orientadas a cambios de conductas y mejores condiciones de vida para estas personas (Acuña y Bulit Goñi, 20I0; REDI et al., 20I2).

El Estado controla y disciplina el acceso de las personas a los sistemas de producción, consumo y ciudadanía. Desde este punto de vista, la discapacidad se define a partir de la exclusión del sistema productivo y de la expulsión de determinadas corporalidades de la producción de bienes y servicios (Oliver, I998). Este modo de construir socialmente las oportunidades de accesos materiales y simbólicos de las personas con discapacidad, excluidas del mundo del trabajo, supone la construcción de su dependencia y el incremento de la necesidad de cuidados. De allí, que el objetivo de este artículo sea reconstruir las políticas públicas en discapacidad mediante el análisis de sus principales leyes y las condiciones de vida de estas personas. A tal fin se consultó: normativa específica, literatura especializada y datos cuantitativos provenientes de diferentes fuentes secundarias disponibles en Argentina.

\section{Palabras clave}

Discapacidad, derechos, políticas públicas, protección social.

\begin{abstract}
In Argentina, I3 \% of the population has some type of disability, a condition that implies inequalities in the social participation of this group. The current regulatory framework is advanced in this area, although it does not generate actions aimed at changing behavior and better living conditions for these people (Acuña and Bulit Goñi, 20ıо; REDI et al., 2012).

The State controls and disciplines the access of people to production, consumption and citizenship systems. From this point of view, disability is defined from the exclusion of the productive system and from the expulsion of certain corporalities from the production of goods and services (Oliver, I998). This way of socially constructing the opportunities of material and symbolic access for people with disabilities, excluded from the world of work, implies the construction of their dependence and the increase in the need for care. Hence the aim of this paper is to reconstruct public policies on disability by analyzing their main laws and the living conditions of these people. To this end, we consulted: specific regulations, specialized literature and quantitative data from different secondary sources available in Argentina.
\end{abstract}

\section{Keywords}

Disability, rights, public policies, social protection.

\author{
María Pía Venturiello \\ <venturiello@yahoo.com.ar> \\ Consejo de Investigaciones Científicas \\ y Técnicas (CONICET). Universidad \\ de Buenos Aires
}

Para citar:

Venturiello, M. P. (20I7): "Políticas sociales en discapacidad: una aproximación desde las acciones del Estado en Argentina". Revista Española de Discapacidad, 5 (2): I 49-I69.

Doi: <https://doi.org/IO.5569/23405 I04.05.02.08>

Fecha de recepción: 24-02-20I7 Fecha de aceptación: 23-IO-20I7

públicas, protección social. 


\section{Introducción}

La discapacidad, según las concepciones más clásicas sobre el tema, indica un problema de salud e individual, donde la organización social y económica resulta secundaria. Desde un enfoque social, se trata de una cuestión que se dirime en el ámbito público. Estas diferentes posibilidades de interpretación se han visto reflejadas en acciones estatales orientadas tanto a reproducir, o bien, a aminorar, las inequidades que afectan a este colectivo.

En Argentina, el I3 \% de la población tiene algún tipo de discapacidad según el Censo Nacional de Población, Hogares y Viviendas de 20I0 (INDEC, 20I4), condición que implica desigualdades en la participación social de este conjunto. El marco normativo vigente resulta avanzado en la materia aunque no llega a generar acciones orientadas a cambios de conductas y mejores condiciones de vida para estas personas (Acuña y Bulit Goñi, 20IO; REDI et al., 20 2). Las desventajas que afectan a esta población se manifiestan en mayores tasas de pobreza y necesidades sanitarias no satisfechas, menores tasas de empleo y un nivel educativo inferior (OMS y Banco Mundial, 20I I). Esta marginación social y económica constituye una forma injusta de segregación originada en la expulsión de los "cuerpos deficientes" de los espacios productivos con consecuencias equivalentes en los restantes espacios sociales.

Las políticas públicas expresan la capacidad del Estado para articular los intereses de diversos actores sociales. Estos constituyen clases, fracciones de clases, organizaciones y grupos que se definen a partir de su capacidad de identificar objetivos, diseñar un curso de acción y contar con relativa autonomía para implementarlos (Oszlak y O’Donnell, I984; Acuña, et al. 2010). Dar respuestas estatales a las necesidades de las personas con discapacidad supone una acción transversal de los distintos organismos del Estado y requiere de su intervención conjunta e integrada (Acuña et al., 20Io), en articulación con la sociedad civil y el mercado.
La discapacidad puede ser entendida tanto como un problema individual, que requiere ciertas asistencias o como un problema social que merece intervenciones estatales. Las disputas en estas definiciones tienen implicancias prácticas sobre las condiciones de vida y el ejercicio de los derechos de las personas. De acuerdo al "modelo social de la discapacidad", es la sociedad la que debe adaptarse a las necesidades de estas personas para hacer efectivos sus derechos y su inclusión social (Oliver, I998; Barnes, I998). En esta línea, se encuentran las propuestas para políticas públicas que promueven la autonomía personal, mediante una serie de apoyos destinados a reducir la dependencia, basados en el respeto de derechos (Díaz Velázquez, 20I 5 ). Esta mirada se distancia de las políticas asociadas al modelo de la rehabilitación, que la ciñe a un problema personal y familiar sin propiciar las modificaciones del entorno social e institucional necesarias para cumplir con la participación social plena de estas poblaciones.

El Estado controla y disciplina el acceso de las personas a los sistemas de producción, consumo y ciudadanía. Desde este punto de vista, la discapacidad se define a partir de la exclusión del sistema productivo y de la expulsión de determinadas corporalidades de la producción de bienes y servicios (Oliver, I998). De este modo, también se restringe la posibilidad de las personas con discapacidad de acceder a su condición de trabajadores y asalariados, una de las figuras de acceso a la protección social (Velandia y Hernández Jaramillo, 2006). Este modo de construir socialmente las oportunidades de accesos materiales y simbólicos de las personas con discapacidad, excluidas del mundo del trabajo, incluye la construcción de su dependencia y el incremento de la necesidad de cuidados. Las principales normas que se han desarrollado en el tema expresan las diferentes orientaciones en el modo de comprender la discapacidad, de delimitar las responsabilidades sociales y de definir las necesidades y áreas de intervención prioritarias. De allí, que el objetivo de este artículo sea una primera aproximación a reconstruir las políticas públicas en discapacidad mediante el análisis de sus principales leyes y las acciones de 
protección social destinadas a las personas en esta condición.

Para responder los interrogantes de este artículo se revisó la literatura especializada. Para el caso específico de Argentina, las investigaciones en el tema aún constituyen un área con desarrollo incipiente. En cuanto a la normativa específica, se seleccionaron las leyes marco a nivel nacional que constituyen las principales referencias en el tema en la atención integral de la población con discapacidad. Para su análisis, se contemplaron las siguientes dimensiones: año de creación; población objetivo; objetivos y alcances jurisdiccional; tipo de documento; perspectiva sobre la discapacidad (asistencialista o de derechos), adecuación o no a la Convención sobre los Derechos de las Personas con Discapacidad; datos relevantes sobre el contexto histórico de su creación. Las fuentes estadísticas consultadas e incorporadas para ofrecer datos contextuales son las existentes a nivel nacional. Por un lado, la más actualizada que constituye el Censo Nacional de Población de Hogares y Viviendas de 2010 (Cen 20I0) y, por otro lado, la Primera Encuesta Nacional de Discapacidad de $2002 / 2003^{\mathrm{I}}$ ambos producidos por el Instituto Nacional de Estadísticas y Censos (INDEC).

La línea argumental del texto plantea, luego de esta introducción, una primera sección acerca de las políticas sociales en general, un segundo apartado aborda la ley marco sobre discapacidad en Argentina, seguido de la presencia del enfoque de derechos en la normativa. A continuación se presentan algunos aspectos relativos a las condiciones de vida a partir de los datos del Cen 20 io y se describen las características de protección social de esta población. Por último, se esbozan las consideraciones finales.

I. Argentina también cuenta con datos sociodemográficos y económicos nacionales producidos por el Servicio Nacional de Rehabilitación, pero solo abarca a la población que ha certificado su discapacidad. Otra fuente relevante para la Ciudad de Buenos Aires, lo constituye el Módulo de discapacidad 20 I I de la Encuesta Anual de Hogares producida por la Dirección de Estadística y Censos de la Ciudad de Buenos Aires.
2. Políticas sociales: principales actores

Argentina constituye uno de los países latinoamericanos donde el estado de bienestar tuvo una gran expansión desde mediados del siglo XX mediante la extensión de la cobertura del sistema previsional; el desarrollo de la seguridad social y los subsistemas de salud denominados obras sociales, junto con el desarrollo de la infraestructura en esa área y la de educación, así como la expansión de la política habitacional (Isuani, 2009). Sin embargo, las políticas neoliberales de los años 90 que derivaron en la crisis institucional y económica de 2001/2002- redujeron esas prestaciones y potenciaron el rechazo a las grandes políticas institucionales en materia de desarrollo social (Danani, 20I6). Este contexto, dio origen a la nueva cuestión social marcada por la exclusión socioeconómica de la población desocupada o en situación de precarización laboral (Isuani, 2009). Como respuesta a este contexto, desde el 2003 la intervención estatal en el campo del bienestar social fue consolidándose paulatinamente en dos grandes líneas de políticas públicas. Por un lado, a partir de la recuperación de la actividad económica, se generaron medidas destinadas a los trabajadores asalariados formales; por otro lado, un conjunto de programas sociales fueron encargados de abordar en el corto plazo las situaciones de las personas en condición de pobreza y la falta de empleo que aparentaban ser transitorias, bajo la forma de un conjunto de medidas destinadas a los sectores no integrados al mercado laboral (Arcidiacono, 20I2). Entre estas acciones se destacan: la reconstitución de rasgos más ampliamente solidarios y con mayor capacidad redistributiva en el caso del Sistema Previsional al incorporar beneficiarios de forma masiva y otorgar movilidad de los haberes; así como la implementación de variados mecanismos de protección para niños, niñas y adolescentes (Danani, 2016).

En este marco general de políticas sociales en el país, las acciones públicas en relación a la discapacidad han sido históricamente escasas, 
aisladas y vinculadas a la rehabilitación y la salud. En la actualidad, los principales actores públicos y de la sociedad civil en el campo de la discapacidad coinciden en la falta de políticas públicas en el tema y la falta de articulación entre los distintos organismos del Estado. Indican la ausencia de planificación y estrategias para desarrollar acciones estatales y una agenda común tanto estatal como por parte de las organizaciones de la sociedad civil. Del mismo modo, se cuestionan las iniciativas aisladas que no convergen en la creación de mecanismos que de manera global aseguren el acceso a derechos y un alcance a la totalidad de la población con discapacidad, agravado por la ausencia de organismos de control. Entre los desafíos que señalan la importancia de ir más allá de las acciones de articulación aisladas y sin continuidad en el tiempo que se llevan adelante entre diferentes organismos públicos entre sí y de la sociedad civil, para consolidar una política transversal (FOCO/CEIPSU, 20I3).

El Estado cuenta con el poder para crear una trama institucional que permite establecer las reglas de juego para la confrontación y los acuerdos entre las distintas fuerzas sociales. En este sentido, se constituye en el principal interlocutor de los distintos actores, por su capacidad de normalizar prácticas, organizaciones y recursos (Belmartino, 2009). Asimismo, mediante las políticas sociales, el Estado actúa en su doble rol de proteger y controlar a los sujetos. Su función de reproductor de lo social: de la vida, de la fuerza de trabajo y de las pautas básicas que orientan las prácticas sociales se relaciona con su capacidad de normatizar y normalizar. Las políticas de Estado refieren al modo en que la cuestión social se convierte en una cuestión de Estado y en políticas sectoriales. El Estado define los problemas sociales, los sujetos merecedores de sus intervenciones, el reconocimiento de unas necesidades y no de otras, así como la delimitación de su propia responsabilidad (Grassi et al., I994). Esto indica que las políticas sociales deben abordarse como la consecuencia del vínculo entre, por un lado, las demandas y presiones sociales $y$, por otro lado, las ideas que imparten los cuerpos profesionales que trabajan al interior de las estructuras estatales. Para reconstruir el entramado de intereses que influyen en la delimitación de esas políticas sociales se requiere conjugar los aspectos aludidos con su contexto histórico, político y social (Ramacciotti, 2010).

La construcción de las políticas sociales en discapacidad está condicionada por los problemas para administrar y planificar acciones continuadas y por las dificultades que encuentran las personas con discapacidad para constituirse en un actor colectivo que articule demandas y ejerza presión. La coordinación de las necesidades y los reclamos se torna más complicada por la heterogeneidad en los tipos de discapacidad, lo que conlleva a una fragmentación en las iniciativas, a una competencia por recursos escasos y a reproducir las lógicas de exclusión de la sociedad hacia este colectivo. Estas características propias de los organismos de la sociedad civil se replican en los organismos del Estado a los que tienen mayor llegada, generando respuestas políticas igualmente parciales y heterogéneas (Acuña et al., 2010).

Pese a que el marco legal vigente sobre discapacidad se encuentra en un estado avanzado respecto del reconocimiento de derechos, éstos no se hacen efectivos a causa del alto incumplimiento de estas leyes (Garcilazo, 2006; Fiamberti, 2008; Fara, 2010). Esas normas/reglamentaciones constituyen el resultado de pugnas de intereses entre los que se encuentran los organismos de la sociedad civil (ONG de servicios y defensa de derechos de las personas con discapacidad), como los organismos del Estado (secretarías y ministerios abocados específicamente a la discapacidad o a políticas sociales que las contemplen) y el mercado (empresas en su rol de empleadores, prestadoras de servicios, prepagas de servicios de salud y obras sociales).

Esta desarticulación y dispersión requiere ser revertida para lograr la universalidad inclusiva de las políticas sociales como un valor que debe ser sostenido. Se trata de atender los bienes y dinámicas que promueven y protegen las 
diferentes políticas específicas y que conducen al bienestar como un bien público, es decir, para todas las personas (Fantova, 20I4). En el caso de las personas con discapacidad, uno de los aspectos nodales para favorecer su inclusión es revertir su expulsión del mercado laboral. En tal sentido, las principales dimensiones de la exclusión se refieren al empleo, consumo, participación política, educación, vivienda, salud, conflicto social y aislamiento social (Anaut y Arza, 20I 5). Esto señala la importancia de avanzar en políticas para este sector, a fin de garantizar su inserción mediante el empleo y otras vías que garanticen su inclusión y protección social. Para el análisis del marco de las políticas sociales en discapacidad en Argentina, a continuación se presentan sus principales leyes.

3. Principales lineamientos del sistema de protección integral de las personas con discapacidad

El modo en que se conceptualiza la discapacidad orienta los modos de intervención del Estado en el tema. En Argentina, el marco normativo vigente sobre discapacidad parte de diferentes perspectivas conceptuales que se desarrollaron en el tiempo, que suelen referirse al modelo médico o modelo social, con presencia cada vez mayor del enfoque de derechos, más acorde al segundo.

Las primeras leyes que se han ocupado de las personas con discapacidad son de principios de siglo XX y legislan sobre aspectos parciales de problemas específicos de personas con discapacidad visual. Hacia I98 I se crea el "Sistema de protección integral de las personas discapacitadas" mediante la Ley $22.43 \mathrm{I}^{2}$. Esta propuesta constituye el principal esquema de respuesta estatal a las necesidades de asistencia, prevención, trabajo, educación, salud y accesibilidad de la población con discapacidad.

2. Su Decreto Reglamentario es $498 / 83$.
La ley establece en qué aspectos se prestará protección a la población con discapacidad por parte del Estado. El artículo 4 indica que deben ser garantizados los servicios de: a) Rehabilitación integral b) Formación laboral o profesional c) Préstamos y subsidios destinados a facilitar su actividad laboral o intelectual d) Regímenes diferenciales de seguridad social e) Escolarización en establecimientos comunes con los apoyos necesarios previstos gratuitamente, $o$ en establecimientos especiales f) Orientación o promoción individual, familiar y social.

Desde su sanción se han presentado modificaciones en algunos artículos y se desarrollaron nuevas normativas que resultan en un conjunto de leyes sobre discapacidad donde conviven diversas perspectivas sobre el tema (Fara, 2010).

Según esta ley es "discapacitada”: "toda persona que padezca una alteración funcional permanente o prolongada, física o mental, que en relación a su edad y medio social implique desventajas considerables para su integración familiar, social, educacional o laboral". Aquí se señala que la alteración funcional genera desventajas en la integración, para lo cual la ley ofrece la protección integral cuyo propósito es: "neutralizar la desventaja que la discapacidad les provoca y les den oportunidad, mediante su esfuerzo, de desempeñar en la comunidad un rol equivalente al que ejercen las personas normales". De este modo, su destino es aminorar las consecuencias que la alteración física o mental conlleva a la persona y el impacto que genera en su inserción social.

Asimismo, se menciona una asociación entre la discapacidad y la desviación de la normalidad. El uso del concepto de normalidad-anormalidad concibe en su enunciación y performatividad una forma de violencia simbólica sobre los "discapacitados", aquellos que se alejan de parámetros físicos y de los comportamientos definidos socialmente como útiles y valiosos. Se señala, además, la imposibilidad de integrarse a su comunidad en el rol equivalente a su edad y medio social como rasgo personal y parte de esa anomalía. En este sentido, la definición que 
brinda la ley, a la vez que reconoce necesidades y ofrece atenciones en rehabilitación, formación y subsidios para ayudar al desempeño laboral reproduce un modo de control social. Afectados por las desventajas que resultan de la discapacidad, la integración posible se presenta como una aproximación a la vida "normal". La discapacidad aparece en esta declaración como un problema de origen biológico, cuyas consecuencias sociales la ley contribuye a reducir mediante las ayudas en servicios de salud, educación y accesibilidad. Esta protección se brinda a la vez que se destaca como condición para la integración la importancia del esfuerzo que la persona debe realizar para desempeñarse en la sociedad. Tal condición expresa el sentido que adquiere la discapacidad para el modelo rehabilitador, según el cual la recuperación física y la integración social constituyen un problema y una responsabilidad del individuo. Desde esta mirada se ignoran los condicionantes sociales que conducen a vivencias discapacitantes, las cuales conforman unas condiciones de posibilidad restringidas en las que se plasman los esfuerzos de superación personal. Esta noción voluntarista expresa los valores extendidos dentro de las instituciones de rehabilitación de la época, caracterizadas por ofrecer integración social mediante las competencias deportivas (Ferrante, 20I4).

En efecto, durante el período de sanción de la ley, en las luchas por la definición de la discapacidad se observa la preeminencia de un enfoque médico-asistencial, aun cuando habían llegado a construirse otras nociones que desafiaban esta mirada. Varios estudios dan cuenta de las demandas por el acceso al trabajo de movimientos políticos de personas con discapacidad en años previos a la sanción de esta normativa. En efecto, estas ideas se vieron expresadas en la Ley 20.923 "Comisión Nacional del Discapacitado", sancionada en 1974 , que no llegó a reglamentarse y fue derogada por la dictadura militar. Este tipo de enfoques implicaban un desafío a la noción asistencialista y dependiente de la discapacidad, que era la más extendida (Testa, 20I I; Joly y Venturiello, 2OI2; Ferrante, 20I4). Años más tarde al momento de sancionar la Ley 22.43 I estas voces no contaron con un contexto democrático de expresión. Los canales institucionales de participación política y social se encontraban clausurados por la dictadura militar. De este modo, antes que el resultado de demandas sociales locales, esta norma responde a la movilización internacional de las PCD y a intereses personales y familiares de algunos funcionarios con peso en el gobierno militar, lo cual cercena su capacidad de expresar una discusión plural y democrática (Fara, 20IO; Joly y Venturiello, 20I2).

Los marcos legales son el resultado de los contextos institucionales y políticos, donde existen tensiones de intereses que, en el caso analizado, dio prioridad a las definiciones internacionales promovidas en torno a la declaración del Año Internacional del Impedido en I98I. Este fue declarado junto a un plan de acción por parte de la Naciones Unidas y su enfoque contempla a los impedimentos como resultado de la relación entre la persona y su medio (Pantano, I993).

Las propuestas de los organismos internacionales usualmente tienen efectos ambivalentes a escala nacional (Bregain, 20I4). Por un lado, brindan un punto de apoyo para las movilizaciones políticas de algunos actores locales, pero por otro, tienden a reducir el alcance de las utopías y alternativas imaginadas por éstos de acuerdo con las soluciones políticas “aceptables” desde los intereses de los agentes más influyentes en la escala internacional (Bregain, 20I4). Las miradas críticas hacia el modelo médico refieren a que las clásicas conceptualizaciones de estos organismos, orientadas a destacar la interacción de la personas con las barreras sociales, continúan destacando los problemas de salud e individuales en el origen de la discapacidad en detrimento de los aspectos contextuales (Barnes, 2009). Como resultado de este proceso, la normativa expresa un sentido de la vida de la personas con discapacidad supeditado a su rehabilitación: a través de lo médico asistencial, el trabajo protegido y la educación especial (Fara, 20I0).

La variedad de temas que aborda esta ley implica un avance en la inclusión en el terreno público 
de las necesidades de esta población referidas a rehabilitación, salud, trabajo protegido y educación. Ello da cuenta de una mirada transversal sobre la discapacidad y las necesidades que deben ser atendidas para abordar el tema. Sin embargo, quedan por fuera de los reconocimientos de derechos de esta legislación las demandas referidas a la inserción laboral de estas personas. Tales reclamos eran impulsados por los colectivos organizados de personas con discapacidad. Su petición implicaba trascender el lugar pasivo que les otorgaba el asistencialismo como sujetos dependientes y conformarse como sujetos con derecho a trabajar (Joly y Venturiello, 20I2). Esta demanda es significativa en tanto desafía el sentido de la discapacidad como condición que imposibilita la inserción en el sistema productivo. Al buscar revertir esta expulsión cuestiona en un mismo movimiento la definición de discapacidad como "incapaz de trabajar" y el funcionamiento del modo de producción capitalista. Como repuesta a la necesidad de inserción laboral, la Ley 22.43 I establece talleres protegidos de producción y el apoyo a la labor de las personas discapacitadas a través del régimen de trabajo a domicilio. Asimismo, el artículo número 8 obliga a los organismos estatales a emplear una cuota del $4 \%$ de personas discapacitadas que reúnan condiciones de idoneidad para el cargo. Aun cuando esto implica un reconocimiento parcial, el avance que representa la existencia de una cuota de empleo de personas con discapacidad solo involucra al sector público y no incluye en esta responsabilidad al sector privado. De este modo, se reproduce un mecanismo de "exclusión incluyente" que reflejan las políticas compensatorias para personas con discapacidad, las cuales suponen su inclusión en circuitos institucionales específicos a la vez que esconden, por estar naturalizada, su exclusión masiva del sistema productivo (Vallejos, 2009). De allí que desde esta perspectiva se perpetúe el lugar de asistencialismo, aún con el reconocimiento de necesidades que deben ser atendidas. El enfoque de derechos, que se presenta como un marco conceptual superador, avanza sobre el modo de concebir las necesidades de las personas con discapacidad ante la ley como derechos que deben ser cumplidos, y no como una asistencia que puede ser ofrecida o negada por parte del Estado.

\section{El enfoque de derechos en discapacidad}

El abordaje de la discapacidad desde un enfoque de derechos se presenta en diversas normativas, aunque no necesariamente se refleja en cambios sustantivos en la vida de estas personas (Díaz Velázquez, 20I 5 ).

En Argentina, adquiere nivel constitucional el reconocimiento de derecho a la igualdad de trato y oportunidades de las personas con discapacidad, junto con otros colectivos considerados desventajados o considerados minorías de acuerdo con las pautas establecidas en los tratados internacionales. Así, el artículo 75 de la Constitución insta a: "Legislar y promover medidas de acción positivas que garanticen la igualdad real de oportunidades y de trato, y el pleno goce y ejercicio de los derechos reconocidos por esta Constitución y por los tratados internacionales vigentes sobre derechos de los niños, las mujeres, los ancianos y las personas con discapacidad (...)" (artículo 75).

Asimismo, Argentina adhiere a varios tratados internacionales, que abogan por la no discriminación y la discriminación positiva como estrategia para reducir las disparidades sociales $^{3}$. Estos abordajes son antecedentes que

3. Retomando la recopilación elaborada por Seda (2015) en esta materia se observa que en I976, con el fin de incrementar la igualdad de oportunidades, prevenir la discapacidad y estimular una mayor participación social, por medio de la sensibilización de la opinión pública, la Asamblea General de la ONU proclamó que I98 I fuera el Año Internacional de los Impedidos, denominación que quedó en desuso por sus connotaciones peyorativas. Otro documento trascendente que menciona la participación plena de las personas con discapacidad en igualdad de condiciones fue el Programa de Acción Mundial para las Personas con Discapacidad aprobado por la Asamblea General de ONU el 3 de diciembre de I982. Este refiere a la importancia de alcanzar mejoras en las condiciones de vida de esta población, en particular en cuanto a su situación social y económica. Un año después, en I983, en la Conferencia General de la Organización Internacional del Trabajo (OIT) se aprobó el Convenio I 59, que plantea la Readaptación Profesional y Empleo de Personas Inválidas (Seda, $2015)$.Un antecedente adicional que aporta una propuesta transversal lo constituye la Declaración de Cartagena de Indias sobre Políticas Integrales para las Personas con Discapacidad en el Área Iberoamericana. Los Estados firmantes, entre ellos la Argentina, se comprometen a diseñar y ejecutar políticas 
se plasman en la legislación nacional acerca de la atención de necesidades de esta población según se observó en apartados anteriores, aún con reminiscencias del modelo de rehabilitación. Sin embargo, el documento internacional más avanzado en la materia, lo constituye la Convención Internacional sobre los Derechos de las Personas con Discapacidad declarada por las Naciones Unidas en 2006 y a la que Argentina se adhiere en 2008 con carácter facultativo.

Entre las primeras características a destacar de esta legislación, se encuentra que a diferencia de otros textos, la definición que plantea acerca de la discapacidad se distingue por la ausencia de connotaciones negativas, como por ejemplo, sostener una carga valorativa de lástima o piedad. En su artículo I indica que: "Las personas con discapacidad incluyen a aquellas que tengan deficiencias físicas, mentales, intelectuales o sensoriales a largo plazo que, al interactuar con diversas barreras, puedan impedir su participación plena y efectiva en la sociedad, en igualdad de condiciones con las demás". Según se observa, incorpora un enfoque dinámico que posibilita adaptaciones a lo largo del tiempo y de acuerdo a los contextos socioeconómicos, mientras destaca la interacción con distintas barreras en el origen de la situación de discapacidad (Rosales, 2008; Seda, 20I 5 ). Ello plantea una definición desde la interacción, que incorpora aspectos del modelo social. No obstante, desde las posiciones defensoras de este modelo más críticas, se sostiene que persiste el marco conceptual del modelo biomédico al definir a la discapacidad desde la deficiencia (Barnes, 2009).

A pesar de las disputas mencionadas acerca del origen y los alcances de la definición, puede sostenerse que este documento y su énfasis en los derechos plantea un paradigma que en sus usos

integrales tendentes a prevenir deficiencias, pero una vez que se hubieran consumado, evitar que tengan consecuencias físicas, psicológicas y sociales negativas. Asimismo, se enuncian diferentes obligaciones estatales acerca de medidas específicas de rehabilitación y atención integral y la disponibilidad de recursos y servicios. En su texto se presentan conceptos tales como no discriminación, vida independiente, autonomía, participación, respeto al propio contexto y vida digna. políticos, jurídicos e institucionales promueve una alternativa a la mirada asistencialista y estigmatizante que profundiza las propuestas de las normativas precedentes por su capacidad de divulgación y estatuto de Convención. De acuerdo con Seda (2015), en Argentina el reconocimiento de derechos en términos jurídicos cuenta con antecedentes para los cuales la Convención implica más una continuidad que un acontecimiento disruptivo en la adopción de medidas. La jurisprudencia sobre discapacidad indica que no se ha sufrido un cambio abrupto a partir de la sanción de la Convención sino que los avances más importantes se consolidan a partir de la reforma constitucional de 1994 y la sanción de normas sobre financiamiento de prestaciones, entre las que se destaca la Ley 24.90I (sancionada en el año I997). En tal sentido, el avance más trascendente de esta normativa internacional la constituye su función de catalizador político y su rol simbólico como emblema del colectivo de personas con discapacidad, antes que como quiebre en el ejercicio jurídico (Seda, 20I5).

En esta línea, un estudio que indagó sobre la mirada de los principales actores sociales en discapacidad, quienes sí perciben a la Convención como un hito plantean que la nueva legislación sirvió de marco para el desarrollo y gestión de una serie de acciones. Estas abarcan desde capacitaciones para difundir la Convención; creación de nuevos organismos como observatorios; producción de materiales accesibles para la inclusión y educación; la realización de espacios que permiten generar conciencia sobre los derechos de las personas con discapacidad, su independencia, autonomía, salud sexual y reproductiva; trabajar sobre su participación política, como el voto accesible; hasta la incidencia en nuevas leyes, como la ley de medios con el subtitulado en TV y la lengua de señas (FOCO/CEIPSU, 20I3).

Así, el valor político de la Convención reside en la intención de profundizar y extender en todas las leyes el enfoque de derechos en discapacidad. El sentido transformador de esta perspectiva se encuentra en el desplazamiento y cambio de estatus de la consideración estatal sobre 
determinados sectores marginales. Estos pasan de ser objeto del asistencialismo, para adquirir el rango de titulares de derechos; de este modo, se genera una nueva distribución de derechos y responsabilidades hacia el Estado. Con este fin se desarrollan mecanismos para el otorgamiento de poder a los sectores postergados y excluidos que supone la responsabilidad del Estado, la igualdad, la no discriminación y la participación social (Abramovich, 2006). Cuando las normativas sobre discapacidad adoptan este enfoque se concibe a las personas con discapacidad como ciudadanos activos, víctimas de las desigualdades generadas por el sistema y no por sus condiciones individuales, dimensión clave en el reconocimiento de la ciudadanía plena de esta población (Díaz Velázquez, 2016). Esta perspectiva, que tiene impactos legislativos y burocráticos, se contradice en términos conceptuales con la noción de discapacidad como problema privado, donde el Estado brinda una asistencia a quienes no pueden valerse por medios propios o familiares. No obstante, su relación con prácticas y ejercicios concretos que modifican la situación efectiva de las personas con discapacidad se encuentra matizada por dificultades culturales e institucionales en torno a la modificación de prácticas arraigadas que responden a una actitud que segrega y medicaliza a las personas con discapacidad (REDI et al., 2012).

El concepto de los derechos humanos no solo se entiende como un límite a la opresión, sino también como un programa que puede guiar las políticas públicas de los Estados orientadas al fortalecimiento de las instituciones democráticas (Abramovich, 2006). Sin embargo, la perspectiva de derechos encuentra sus límites en las dificultades para convertir los preceptos de no discriminación y opresión en políticas estatales integrales. Crear instituciones y mecanismos que funcionen de acuerdo a estos parámetros depende de las idiosincrasias institucionales, las luchas de intereses, y la estructura socioeconómica de cada sociedad. Su implementación requiere formar parte en la vida cotidiana de ciudadanos encarnados en sujetos particulares, dentro de una cultura, sociedad y economía específicas.
En lo que respecta a las personas con discapacidad, el reconocimiento de sus derechos requiere ser concebido dentro de una sociedad que los segrega y estigmatiza y a su vez excluye del sistema productivo (Kipen y Vallejos, 2009). Así, el reconocimiento legal se da en un contexto cultural donde los valores predominantes se vinculan a las categorías de normalidad y anormalidad que implican su desmedro simbólico y material.

\section{Condiciones de vida de la población con discapacidad}

Para describir las condiciones de vida de esta población se utilizaron los datos del Censo Nacional de Población, Hogares y Viviendas 20Io (Cen 20IO) (INDEC, 20I4). En esta medición, el diseño conceptual se basó en el enfoque bio-psicosocial de la discapacidad que propone la Clasificación Internacional del Funcionamiento, de la Discapacidad y de la Salud (CIF) del año 200I. Los resultados del Cen 20 Io refieren a las dificultades o limitaciones permanentes (físicas y/o mentales) que la población declaró en el cuestionario censal. Aquí se utiliza el término personas con discapacidad para agilizar la lectura. La unidad de análisis "Población", se ubicó en el cuestionario ampliado que se aplicó al ıo०\% de las viviendas en localidades de menos de 50 mil habitantes y a un 10\% de las viviendas de las localidades de más de 50 mil habitantes. A continuación se presentan datos generales sociodemográficos sobre sexo y edad, educación, condiciones de vida, ocupación, salud y previsión social. 
- Sexo y edad

Cuadro 1. Población en viviendas particulares, población con discapacidad y prevalencia de la discapacidad, por sexo. Total del país (2010)

\begin{tabular}{|l|c|c|c|}
\hline & $\begin{array}{c}\text { Población } \\
\text { total en } \\
\text { viviendas } \\
\text { particulares }\end{array}$ & $\begin{array}{c}\text { Población } \\
\text { con } \\
\text { discapacidad }\end{array}$ & Prevalencia \\
\hline Total & 39.671 .131 & 5.114 .190 & $12,90 \%$ \\
\hline Varones & 19.276 .217 & 2.263 .175 & $11,70 \%$ \\
\hline Mujeres & 20.394 .914 & 2.851 .015 & $14 \%$ \\
\hline
\end{tabular}

Fuente: INDEC. Censo Nacional de Población, Hogares y Viviendas 2010.

Según el Censo 2010, 5.I I4.I90 personas se encuentran en condición de discapacidad y su prevalencia es mayor entre las mujeres ( $14 \%$ )

Cuadro 2. Prevalencia de la discapacidad en viviendas particulares por grupo de edad. Total del país (2010). Porcentajes

\begin{tabular}{|l|c|}
\hline Grupo de edad & Prevalencia en $\%$ \\
\hline $0-4$ & 2,5 \\
\hline $5-9$ & 4,8 \\
\hline $10-14$ & 6 \\
\hline $15-19$ & 5,8 \\
\hline $20-24$ & 6 \\
\hline $25-29$ & 6,5 \\
\hline $30-34$ & 7 \\
\hline $35-39$ & 8 \\
\hline $40-44$ & 10,9 \\
\hline $45-49$ & 17,1 \\
\hline $50-54$ & 22,3 \\
\hline $55-59$ & 25,8 \\
\hline $60-64$ & 28,2 \\
\hline $65-69$ & 31,4 \\
\hline $70-74$ & 36,7 \\
\hline $75-79$ & 43,6 \\
\hline 80 y más & 56,4 \\
\hline Total & 12,9 \\
\hline & \\
\hline
\end{tabular}

Fuente: elaboración propia en base a Censo Nacional de Población, Hogares y Viviendas 20I0. INDEC que entre los varones ( $12 \%$ ). Con respecto a la prevalencia de la discapacidad según grupos etarios (Cuadro 2), se observa que aumenta a medida que envejece la población.

\section{- Educación}

Los datos sobre educación comparables entre la población con discapacidad y la población total refieren a la asistencia a un establecimiento educativo. Según se puede observar, las personas con discapacidad asistían al sistema educativo al momento de realizar la encuesta en menor medida ( $14,4 \%$ ) que la población total $(32,3 \%)$. Sin embargo, la proporción de personas con discapacidad que asistieron en el pasado es mayor. Esto refleja las desigualdades no solo en el acceso a los establecimientos de formación, sino también la dificultad para permanecer en el ciclo escolar en los diferentes niveles. Adicionalmente, las personas con discapacidad que nunca asistieron a un establecimiento educativo superan el $5 \%$, mientras que en la población total se reduce al $3 \%$ (Cuadro 3 ).

\section{Cuadro 3. Población total de 3 años y más y} población de 3 años y más con discapacidad por condición de asistencia escolar en Argentina (2010). Porcentajes

\begin{tabular}{|l|c|c|}
\hline $\begin{array}{l}\text { Condición } \\
\text { asistencia } \\
\text { escolar }\end{array}$ & $\begin{array}{c}\text { Población } \\
\text { total }\end{array}$ & $\begin{array}{c}\text { Población con } \\
\text { discapacidad }\end{array}$ \\
\hline Asiste & 32,3 & 14,4 \\
\hline Asistió & 64,7 & 80,4 \\
\hline Nunca asistió & 3 & 5,2 \\
\hline Total & 100 & 100 \\
\hline
\end{tabular}

Fuente: elaboración propia en base a Censo Nacional de Población, Hogares y Viviendas 20Io. INDEC. 


\section{- Condiciones de vida}

Este apartado refiere al acceso de la población con discapacidad a los servicios básicos como: condiciones de vivienda, servicio de agua potable y de cloacas y su relación con la condición de pobreza.

Uno de los indicadores para evaluar y diagnosticar el estado de situación de las viviendas es INMAT. Este instrumento las clasifica en aceptables, recuperables e irrecuperables, de acuerdo con las modificaciones que requiera en sus materiales para satisfacer las necesidades del hogar. Según la medición del Cen 20IO se observa que las viviendas aceptables, aquellas que por la calidad de los materiales, no necesitan reparación para satisfacer las necesidades del hogar, son menos frecuentes en los hogares donde hay alguna persona con discapacidad en comparación con el total de viviendas $(58,2 \%$ vs. $62,6 \%)$. Por el contrario, es mayor el porcentaje de viviendas que pueden considerarse precarias en aquellas donde habita al menos una persona con discapacidad (Cuadro 4). De allí que las condiciones de vida de esta población evidencien la vinculación entre discapacidad y pobreza y la multiplicación de dificultades que supone para el desenvolvimiento diario de estas personas. Así, estos datos permiten señalar la interrelación discapacidadpobreza de carácter bidireccional (Anaut y Arza, 20I 5), puesto esas condiciones habitacionales

\begin{tabular}{l}
\hline $\begin{array}{l}\text { Cuadro 4. Total de viviendas particulares y } \\
\text { viviendas con al menos una persona con } \\
\text { discapacidad en Argentina (2010). Porcentajes }\end{array}$ \\
\begin{tabular}{|l|c|c|}
\hline $\begin{array}{l}\text { Calidad de } \\
\text { vivienda }\end{array}$ & $\begin{array}{c}\text { Total de } \\
\text { viviendas } \\
\text { particulares }\end{array}$ & $\begin{array}{c}\text { Viviendas } \\
\text { particulares con } \\
\text { al menos una } \\
\text { persona con } \\
\text { discapacidad }\end{array}$ \\
\hline Aceptable & 62,6 & 58,2 \\
\hline Recuperable & 33,3 & 37,2 \\
\hline Irrecuperable & 4,1 & 4,6 \\
\hline Total & 100 & 100 \\
\hline
\end{tabular}
\end{tabular}

Fuente: elaboración propia en base a Censo Nacional de Población, Hogares y Viviendas 20ro. INDEC. empeoran la calidad de vida de esta población profundizando su discapacitación.

Otros indicadores también reflejan la accesibilidad en los espacios para la vida cotidiana de las personas con discapacidad y su relación con los servicios básicos. En cuanto a la provisión del agua, el $97 \%$ de las personas que habita en viviendas con al menos una personas con discapacidad cuenta con la posibilidad de acceder a este servicio dentro del terreno donde se encuentra la vivienda. Mientras en la población total, este porcentaje llega a casi el $98 \%$. Asimismo, el 48,1 \% de la población con discapacidad en viviendas particulares tiene desagüe del inodoro a cloaca, en tanto que en la población total alcanza al 48,8\% (INDEC, 20I4).

Finalmente, el concepto de Necesidades Básicas Insatisfechas ${ }^{4}$ (NBI) permite la delimitar grupos de pobreza estructural al identificar dimensiones de carencias absolutas y define a la pobreza a partir de un cúmulo de privaciones materiales esenciales. Los hogares con NBI constituyen un mayor porcentaje en los hogares donde vive al menos una persona con discapacidad en comparación con el total de hogares ( I I, I \% vs. 9, I \%) (Cuadro 5).

4. Se consideran hogares con NBI a aquellos que presentan al menos una de las siguientes privaciones:

- NBI I. Vivienda: es el tipo de vivienda que habitan los hogares que moran en habitaciones de inquilinato, hotel o pensión, viviendas no destinadas a fines habitacionales, viviendas precarias y otro tipo de vivienda. Se excluye a las viviendas tipo casa, departamento y rancho.

- NBI 2. Condiciones sanitarias: hogares que no poseen retrete.

- NBI 3. Hacinamiento: es la relación entre la cantidad total de miembros del hogar y la cantidad de habitaciones de uso exclusivo del hogar. Operacionalmente se considera que existe hacinamiento crítico cuando en el hogar hay más de tres personas por cuarto.

- NBI 4. Asistencia escolar: hogares que tienen al menos un niño en edad escolar ( 6 a I 2 años) que no asiste a la escuela.

- NBI 5. Capacidad de subsistencia: hogares que tienen cuatro o más personas por miembro ocupado y que tienen un jefe que no ha completado el tercer grado de escolaridad primaria. 


\begin{tabular}{l}
\hline $\begin{array}{l}\text { Cuadro 5. Total de hogares y hogares con al } \\
\text { menos una persona con discapacidad por } \\
\text { presencia de NBI en Argentina (2010). Porcentajes }\end{array}$ \\
\begin{tabular}{|l|c|}
\hline & $\begin{array}{c}\text { Hogares } \\
\text { con NBI }\end{array}$ \\
\hline Total de hogares & 9,1 \\
\hline $\begin{array}{l}\text { Hogares con al menos una persona } \\
\text { con discapacidad }\end{array}$ & 11,1 \\
\hline
\end{tabular}
\end{tabular}

Fuente: elaboración propia en base a Censo Nacional de Población, Hogares y Viviendas 20Io. INDEC.

\section{- Ocupación}

Las condiciones materiales de las personas con discapacidad se encuentran asociadas a un mercado que los excluye como trabajadores. Las diferentes barreras sociales, culturales y arquitectónicas funcionan desalentando las intenciones de participar en el mercado de trabajo de las personas con discapacidad. Entre las múltiples desigualdades sociales que afronta esta población se observan sus altos niveles de desocupación e inactividad. De acuerdo con el Cen 2010 entre las 4.701 .685 personas con discapacidad de I 4 años o más, existe un alto porcentaje de población inactiva $(52 \%)$, mientras los inactivos en la población total se reducen al 34,4\%. En la misma línea, las tasas de empleo para la población total son superiores a las de la población con discapacidad. Si se observa por grupos de edad las mayores diferencias se ubican entre quienes tienen entre

\begin{tabular}{|c|c|c|}
\hline \multicolumn{3}{|c|}{$\begin{array}{l}\text { Cuadro 6. Población total de } 14 \text { años y más y } \\
\text { población de } 14 \text { años y más con discapacidad } \\
\text { por condición de actividad económica en } \\
\text { Argentina (2010). Porcentajes }\end{array}$} \\
\hline $\begin{array}{l}\text { Condición de } \\
\text { actividad }\end{array}$ & $\begin{array}{l}\text { Población } \\
\text { total }\end{array}$ & $\begin{array}{l}\text { Población con } \\
\text { discapacidad }\end{array}$ \\
\hline $\begin{array}{l}\text { Población } \\
\text { económicamente } \\
\text { activa }\end{array}$ & 65,6 & 47,7 \\
\hline $\begin{array}{l}\text { Población } \\
\text { económicamente } \\
\text { inactiva }\end{array}$ & 34,4 & 52,3 \\
\hline Total & 100 & 100 \\
\hline
\end{tabular}

Fuente: elaboración propia en base a Censo Nacional de Población, Hogares y Viviendas 20I0. INDEC.
35 y 44 años, a favor de la población total (8I \% vs. $67 \%$ ) (Cuadros 6 y 7).

Las personas con discapacidad que trabajan como empleados u obreros $(60 \%)$ son menos que aquellas que cuentan con estos empleos en la población total $(72 \%)$. Como consecuencia, se eleva la proporción de cuentapropistas entre las personas con discapacidad $(27,5 \%)$, condición que incluye un amplio abanico de trabajos, generalmente con menos garantías de estabilidad que los empleos (Cuadro 8). En efecto, la posibilidad de acceder a un trabajo, no alcanza para describir la relación entre empleabilidad y discapacidad, puesto que otras dimensiones refieren a su intensidad y ejercicio a lo largo de todo el año (Barragué, 20I5).

Asimismo, se observa que la proporción de las personas con discapacidad empleadas en el sector privado (69\%) es menor que en la población total $(72 \%)$. Como contrapartida su participación en el sector público $(3$ I \%) es mayor, pese al incumplimiento del cupo laboral para personas con discapacidad en el Estado (Cuadro 9). Esto evidencia las dificultades adicionales de inserción en el sector privado que no está obligado por ley a emplear a esta población. Sobre el tipo de barreras que enfrentan las personas con discapacidad para ser empleadas resulta elocuente una encuesta realizada en España en 2012 por el Instituto Nacional de Estadística según el cual 90,3\%

Cuadro 7. Tasa de empleo de la población total y
de la población con discapacidad por grupo de
edad en Argentina (2010). Porcentajes
\begin{tabular}{|l|c|c|}
\hline Grupo de edad & $\begin{array}{c}\text { Población } \\
\text { total }\end{array}$ & $\begin{array}{c}\text { Poblacion con } \\
\text { discapacidad }\end{array}$ \\
\hline $14-24$ & 44,8 & 38,4 \\
\hline $25-34$ & 77,5 & 64,3 \\
\hline $35-44$ & 80,6 & 67 \\
\hline $45-54$ & 78,2 & 66,5 \\
\hline $55-64$ & 65 & 54,2 \\
\hline 65 años y más & 25,3 & 18,3 \\
\hline
\end{tabular}

Fuente: elaboración propia en base a Censo Nacional de Población, Hogares y Viviendas 20I0. INDEC. 
de las personas con discapacidad, indican algún tipo de barrera para acceder a un empleo adecuado entre las que se destacan la falta de oportunidades, los motivos de salud y la falta de cualificaciones o experiencia (SIIS Centro de Documentación y Estudios, 20I4).

\begin{tabular}{|c|c|c|}
\hline \multicolumn{3}{|c|}{$\begin{array}{l}\text { Cuadro 8. Población de } 14 \text { años y más ocupada } \\
\text { y población de } 14 \text { años ocupada y más con } \\
\text { discapacidad por condición de actividad } \\
\text { económica (2010). Porcentajes }\end{array}$} \\
\hline $\begin{array}{l}\text { Categoría } \\
\text { ocupacional }\end{array}$ & $\begin{array}{l}\text { Población } \\
\text { total }\end{array}$ & $\begin{array}{l}\text { Población con } \\
\text { discapacidad }\end{array}$ \\
\hline $\begin{array}{l}\text { Obrero o } \\
\text { empleado }\end{array}$ & 71,7 & 60 \\
\hline Patrón & 6,6 & 7,5 \\
\hline Cuentapropista & 18,5 & 27,5 \\
\hline $\begin{array}{l}\text { Trabajadores } \\
\text { familiares }\end{array}$ & 3,1 & 5 \\
\hline Total & 100 & 100 \\
\hline
\end{tabular}

Fuente: elaboración propia en base a Censo Nacional de Población, Hogares y Viviendas 20ı. INDEC.

Cuadro 9. Obreros o empleados por sector laboral: población total y población con dificultad o limitación permanente. Porcentajes

\begin{tabular}{|l|c|c|}
\hline Sector laboral & $\begin{array}{c}\text { Población } \\
\text { total }\end{array}$ & $\begin{array}{c}\text { Población con } \\
\text { discapacidad }\end{array}$ \\
\hline Público & 27,9 & 31,1 \\
\hline Privado & 72,1 & 68,9 \\
\hline Total & 100 & 100 \\
\hline
\end{tabular}

Fuente: elaboración propia en base a Censo Nacional de Población, Hogares y Viviendas 20Io. INDEC.

\section{- $\quad$ Salud}

En Argentina, el sistema de servicios de salud consta de tres subsistemas: público, seguro social y seguro privado. El primero indica las prestaciones y el financiamiento por parte del Estado. El seguro social refiere a la cobertura que obtienen los trabajadores y sus familiares a cargo, mediante la filiación obligatoria a una obra social a la que aportan trabajadores y empleadores. El subsistema privado se caracteriza por la adhesión voluntaria y el pago total de servicios por parte del beneficiario.

En cuanto al acceso a la salud, en el marco de los subsistemas mencionados, casi el $70 \%$ de las personas con discapacidad tiene algún tipo de cobertura en salud, proporción que supera a la población total (64\%) (Cen, 2010). Es posible que esto obedezca al mayor peso de la población adulta mayor en este grupo poblacional, las cuales al contar con jubilación tienen la cobertura correspondiente denominada PAMI, obra social para jubilados.

Entre las personas con discapacidad que declaran tener cobertura, el 92,5\% tiene obra socials, lo que incluye a PAMI.

Aproximadamente el $4 \%$ tiene cobertura por contratación voluntaria y otro $4 \%$ tiene cobertura por programas estatales de salud ${ }^{6}$, ello evidencia que una pequeña proporción puede acceder a servicios de salud prepagos, los cuales indican un mayor potencial económico en esas personas respecto al resto. En la población total quienes acceden a prepagas duplican $(8 \%)$ a las personas con discapacidad y también derivan aportes de sus obras sociales a prepagas en mayor medida que este grupo. Estos datos muestran una segmentación en el tipo de acceso a la salud y en la contratación de servicios prepagos que implican desigualdades (Cuadros IO y II).
5. De este grupo, el 10,5\% deriva sus aportes a una prepaga. Esta opción, implica un costo adicional obligatorio y no todas las personas pueden afrontarlo.

6. Se trata de PROFE, cobertura de salud que incluye todos los servicios detallados en el Plan Médico Obligatorio, que deben tener garantizados la "canasta básica" de prestaciones médicas asistenciales, según la Superintendencia de Servicios de Salud. Sin embargo, debido al desfinanciamiento de este programa, sólo entrega medicamentos y cobertura en el subsistema público. 


\begin{tabular}{l}
\hline $\begin{array}{l}\text { Cuadro 10. Población total y población con } \\
\text { dificultad o limitación permanente por tenencia de } \\
\text { cobertura de salud en Argentina (2010). Porcentajes }\end{array}$ \\
\begin{tabular}{|l|c|c|}
\hline $\begin{array}{l}\text { Cobertura en } \\
\text { salud }\end{array}$ & $\begin{array}{c}\text { Población } \\
\text { total }\end{array}$ & $\begin{array}{c}\text { Población con } \\
\text { discapacidad }\end{array}$ \\
\hline Tiene & 63,9 & 69 \\
\hline No tiene & 36,1 & 31 \\
\hline Total & 100 & 100 \\
\hline
\end{tabular}
\end{tabular}

Fuente: elaboración propia en base a Censo Nacional de Población, Hogares y Viviendas 20ro. INDEC.

Cuadro 11. Población total y población con discapacidad por tipo de cobertura de salud en Argentina (2010). Porcentajes

\begin{tabular}{|l|c|c|}
\hline Cobertura en salud & $\begin{array}{c}\text { Población } \\
\text { total }\end{array}$ & $\begin{array}{c}\text { Población } \\
\text { con } \\
\text { discapacidad }\end{array}$ \\
\hline $\begin{array}{l}\text { Obra social (Incluye } \\
\text { PAMI) }\end{array}$ & 72,6 & 81 \\
\hline $\begin{array}{l}\text { Prepaga a través de } \\
\text { obra social }\end{array}$ & 16,5 & 10,5 \\
\hline $\begin{array}{l}\text { Prepaga a través } \\
\text { de contratación } \\
\text { voluntaria }\end{array}$ & 8,0 & 4,5 \\
\hline $\begin{array}{l}\text { Programas y planes } \\
\text { estatales de salud }\end{array}$ & 2,9 & 4 \\
\hline Total & 100,0 & 100 \\
\hline
\end{tabular}

Fuente: elaboración propia en base a Censo Nacional de Población, Hogares y Viviendas 20ro. INDEC.

Según se mencionó anteriormente, la población con discapacidad se concentra en las edades avanzadas. A partir de los 65 años, en la población total quienes reciben jubilación y/o pensión superan el 90\% (Findling y López, $2015)$. Este alto grado de cobertura forma parte de una política de ampliación jubilatoria para la población en general (con o sin discapacidad) iniciada en 2003. Debido a la concentración de las personas con discapacidad en las edades avanzadas, el $45 \%$ recibe jubilación o pensión mientras que en la población total esta percepción se reduce al I 5 \% (Cuadro I2).

\begin{tabular}{l}
\hline $\begin{array}{l}\text { Cuadro 12. Población total y población con } \\
\text { discapacidad por percepción de jubilación o } \\
\text { pensión en Argentina (2010). Porcentajes }\end{array}$ \\
\begin{tabular}{|l|c|c|}
\hline $\begin{array}{l}\text { Percibe } \\
\text { Jubilación o } \\
\text { pensión }\end{array}$ & $\begin{array}{c}\text { Población } \\
\text { total }\end{array}$ & $\begin{array}{c}\text { Población con } \\
\text { discapacidad }\end{array}$ \\
\hline Sí & 15,2 & 45,3 \\
\hline No & 84,8 & 54,7 \\
\hline Total & 100 & 100 \\
\hline
\end{tabular}
\end{tabular}

Fuente: elaboración propia en base a Censo Nacional de Población, Hogares y Viviendas 20I0. INDEC.

De acuerdo con los datos observados, existen de desigualdades que afectan a la población con discapacidad. Ello evidencia que la ampliación de derechos hacia las personas con discapacidad se encuentra con contradicciones materiales. Así, en cuanto a la expansión de los derechos en materia de discapacidad, se encuentran desigualdades sociales referidas a:

- Menor acceso a establecimientos educativos $(32 \%)$

- $\quad$ i $\%$ de hogares con discapacidad con NBI

- $\quad 52 \%$ de población con discapacidad inactiva

- Menor capacidad de acceso a servicio de salud prepago ( $4 \%$ contratación directa y I० \% con derivación de obra social). Este punto expresa una capacidad económica menor en este grupo que en el total de la población.

A continuación, interesa presentar los principales rasgos de la normativa sobre protección social de esta población, puesto que sufren mayores desventajas y vulnerabilidad social que la población general.

\section{6. ¿Quién es el responsable? Protección social, salud y cuidados}

La forma de organizar el riesgo social de cada Estado influye en las condiciones de vida de 
la población con discapacidad. Según EspingAndersen (2000), la configuración de las instituciones que brindan protección social se corresponden con la forma en que se reparten el riesgo social entre las políticas públicas sociales, el mercado de trabajo y la familia, generando equidad o desigualdades en el acceso. En Argentina, la protección social se organiza principalmente sobre una base contributiva y el sistema de servicios de salud consta de tres subsistemas: público, seguro social y seguro privado. Las discusiones acerca de este sistema de salud refieren a que promueve un acceso a la salud fragmentario, inequitativo y que reproduce desigualdades sociales (Findling, 20I2; Belmartino, 2009).

En este marco, y en conjunto con las normativas específicas y los derechos reconocidos, se configuran las instituciones responsables ante las necesidades de la población con discapacidad.

En I997, se sancionó la Ley 24.90I: "Sistema de prestaciones básicas en habilitación y rehabilitación integral a favor de las personas con discapacidad" que define las prestaciones en prevención, asistencia, promoción y protección, con el objeto de brindar una cobertura integral a las necesidades y requerimientos de esta población. En correspondencia con una política de retracción de garantías sociales ciudadanas propia de la década de los I990, esta ley sostiene una ambigua atribución de responsabilidades hacia el Estado y se focaliza en quienes carecen de recursos. De acuerdo con su artículo 3: "El Estado, a través de sus organismos, prestará a las personas con discapacidad no incluidas dentro del sistema de las obras sociales, en la medida que aquellas o las personas de quienes dependan no puedan afrontarlas, los siguientes servicios". Con esta modificación de la Ley 22.43 I, donde el Estado se constituía en el responsable de atender las necesidades, se presenta a las obras sociales y la medicina prepaga como principales prestadoras, las familias como soporte permanente y, solo en caso de fallar ambas instancias, se recurre al Estado. El resultado de ello es una segmentación de la protección entre quienes están afiliados al sistema de seguridad social por estar incluidos en el mercado de trabajo formal o poseer recursos para acceder a una prepaga, y quienes están desocupados o sus trabajos están dentro del mercado informal y dependen de las prestaciones del Estado. De este modo, la condición de acceso al trabajo formal y las desigualdades socioeconómicas generan inequidades en el acceso a la salud y la protección social. Esto profundiza un proceso de individuación en el que el Estado traspasa su responsabilidad en el sujeto y en las entidades que lo deberían amparar.

En efecto, el gasto social se concentra en el componente contributivo mediante los aportes que realizan los trabajadores, de modo que la condición de asalariado se constituye en un requisito para acceder a los servicios necesarios para la reproducción social de los sujetos (FLACSO, 20I4). Esta condición se constituye en un modo de inequidad social entre las personas que se encuentran desocupadas, inactivas o forman parte del mercado informal, dado que la población destinataria del gasto social se concentra en aquellos que pueden constituirse, mediante el régimen laboral, en contribuyentes. Esto deviene en un problema para quienes, a raíz de la creciente informalidad laboral, quedan por fuera de la posibilidad de ser aportantes y resultan excluidos del sistema contributivo de protección social7. De acuerdo a los datos presentados en la sección anterior, las personas con discapacidad presentan menos posibilidades de acceso a este tipo de cobertura mediante la condición laboral asalariada.

\section{Atención en salud y cuidados}

La atención especializada y la rehabilitación que en muchos casos debe ser permanente se garantiza mediante la obtención del certificado de discapacidad. Este es el requisito para el acceso al mencionado sistema de protección

7. Cabe aclarar que desde 2003 los monotributistas pueden acceder a una obra social. 
integral (Ley 22.43 I) y al sistema de prestaciones básicas (Ley 24.90I), que ayuda ante los tratamientos y cuidados específicos que en muchos casos son prolongados, así como para ciertos apoyos de la vida cotidiana.

La población certificada con discapacidad alcanza a 73 I.745 personas en 2015 (Servicio Nacional de Rehabilitación, 20I6), lo que no abarca al total de la población con discapacidad. En efecto, acceder al trámite de certificación suele implicar un amplio conocimiento, lidiar con trámites burocráticos y contar con redes sociales para llevarlo adelante. La tramitación de esta herramienta legal se encuentra atravesada por controversias teóricas. Por un lado, se afirma que la tramitación de este certificado implica convalidar la noción de discapacidad como etiqueta y estigmatización, por otro lado resulta el modo de acceso a los derechos legalmente reconocidos. Esta dualidad por parte del ejercicio estatal evidencia que el reconocimiento formal de la legislación puede entrar en tensión con las desigualdades en las condiciones de que vida que ese mismo estado perpetua para la población con discapacidad.

Aún contando con el certificado de discapacidad y sus habilitaciones, estudios cualitativos evidencian que los inconvenientes burocráticos y los abordajes fragmentarios del sistema de salud, no alcanzan a responder adecuadamente a las atenciones en salud, físicas y psicológicas de muchas personas con discapacidad y en situación de dependencia (Venturiello, 2016; Findling y López, 20I 5 ). Incluso con las prestadoras prepagas se presentan situaciones litigiosas que demoran la entrega de prótesis, órtesis y asistencias prolongadas. Entre las mayores dificultades de acceso a la salud de las personas con discapacidad se encuentran el financiamiento y la asequibilidad. De acuerdo a la Encuesta Mundial en Salud (2002-2004), en los países de ingresos bajos el factor económico fue el principal motivo por el cual las personas con discapacidad no recibieron la atención en salud que necesitaban (OMS y Banco Mundial, 2OII). El transporte y la accesibilidad de los tratamientos y los profesionales pueden constituirse en otro obstáculo.
En efecto, el acceso a tratamientos adecuados de la salud también se encuentra en estrecha relación con el nivel socioeconómico de las personas con discapacidad. Según la primera Encuesta Nacional de Discapacidad (ENDI) de 2002/2003, se evidenció una importante participación de las familias mediante ayudas instrumentales y financieras para contribuir a la calidad de vida y la atención en salud de las personas con discapacidad. Por ejemplo, respecto de las ayudas técnicas externas que utilizan estas personas y resultan elementos necesarios para desenvolverse en la vida cotidiana, se observa que un poco más de $50 \%$ es cubierto por el presupuesto del hogar. Cabe destacar que este tipo de resoluciones de gestión privada genera una reproducción de las inequidades sociales, ya que entre quienes no utilizan este tipo de ayudas, el 56,4\% declara necesitarlos pero no contar con los recursos económicos para obtenerlas. Asimismo, de las 646.I 24 personas de la muestra que reciben o recibieron tratamiento y rehabilitación casi la tercera parte lo sostiene con presupuesto del hogar $(34,4 \%)$. Nuevamente, entre las 3 I I.2 I 6 personas que no reciben o no recibieron rehabilitación pero expresan necesitarla, los motivos económicos constituyen el principal impedimento. Según esta fuente, la posibilidad de optar por servicios acordes a las necesidades y acceder hasta el lugar del tratamiento está directamente ligada a la posibilidad de pagar por esto. Sumado a ello, el estudio cualitativo de los itinerarios terapéuticos de las personas con discapacidad en busca de su rehabilitación muestra que los incumplimientos de las obras sociales y prepagas en las prestaciones, así como las deficiencias del sistema público, conducen a que los gastos en salud deban asumirse con el presupuesto del hogar (Venturiello, 20I6). En este sentido cabe destacarse, que los itinerarios de las personas con las políticas públicas y las instituciones que las recrean no son homogéneos (Colectivo IOÉ, I998) y las familias construyen varias estrategias para lograr obtener atención en salud.

De acuerdo con lo observado para las personas con discapacidad, los países de la región latinoamericana en la distribución de 
responsabilidades del riesgo social otorgan el protagonismo a las familias, en articulación con la protección social que brinda el Estado. Este modalidad se sostiene en una ideología familista que supone que la consanguineidad y el parentesco son los criterios básicos para las responsabilidades y obligaciones hacia los otros (Jelin, I994).

El desarrollo de la protección social se vincula a las nociones de quienes son los responsables del cuidado de los sujetos y si éste es concebido como un derecho o como una carga familiar. En Argentina, las políticas públicas no expresan un reconocimiento del cuidado como parte de los derechos ciudadanos. La ausencia de políticas públicas en ese sentido deja en manos de las personas, según su disponibilidad de recursos económicos, la posibilidad de cubrir las necesidades del cuidado de familiares y reproduce las desigualdades sociales (Rodríguez Enríquez, 2005).

\section{A modo de cierre}

Este artículo propuso una primera aproximación al análisis de las políticas públicas en discapacidad en Argentina mediante la revisión de sus principales leyes. Se observó que las normas que abarcan varios aspectos de la vida social (salud, rehabilitación, educación, trabajo, entre otros) son la Ley 22.43 I "Sistema de protección integral de las personas con discapacidad", la Ley $24.90 \mathrm{I}$ "Sistema de prestaciones básicas en habilitación y rehabilitación integral a favor de las personas con discapacidad" y la adhesión a la Convención Internacional por los Derechos de las Personas con Discapacidad.

Estas normas que se han sancionado en diversos momentos políticos, donde se encuentran diversas definiciones sobre la discapacidad, avanzan desde un enfoque biomédico hacia uno con énfasis en los derechos. Conforme a los desarrollos conceptuales en el tema a lo largo del tiempo se encontró un desplazamiento desde las nominaciones despectivas que asocian la discapacidad a la anormalidad hacia las que evitan las connotaciones negativas mediante una progresiva preocupación por las barreras sociales. Estas denominaciones responden a las propuestas de un conjunto de actores en disputa que adquieren alternativamente preponderancia en contextos constitucionales diversos y se reflejan en los documentos de los organismos internacionales, los intereses particulares de quienes detentan el poder político y las demandas de los colectivos de derechos de personas con discapacidad.

En relación con la protección social, en el trabajo se observó que gran parte de la población con discapacidad accede a jubilación o pensión y, mediante ello, a cobertura en salud (INDEC, 20I4). Sin embargo, también se observaron desigualdades en el acceso a la salud, la educación y una mayor prevalencia de indicadores de pobreza en esta población.

El análisis de la ley de prestaciones básicas permitió observar la delimitación de los responsables de brindar estos servicios (Ley 24.90I), donde se encontró un señalamiento de las familias y las obras sociales que matizan la responsabilidad del Estado. En este sentido, si bien se ha avanzado en normativas que obligan al Estado a responder a las necesidades de la población con discapacidad mediante la incorporación de adhesiones a tratados internacionales de derechos humanos, la privatización de las responsabilidades conduce a profundizar en las desigualdades estructurales y dificulta el acceso igualitario a los derechos reconocidos.

Tal ambivalencia en el funcionamiento burocrático del Estado parte de su legitimación del modo de producción que construye las "incapacidades" a la vez que reconoce las necesidades y brinda ayudas específicas para favorecer la "integración social" de aquellos que excluye (Vallejos, 2009).

La legislación existente si bien ha incorporado la perspectiva del modelo social, su cumplimiento 
efectivo debe analizarse en un marco institucional donde priman prácticas contradictorias y un contexto de desigualdad social. No obstante, continúa siendo responsabilidad ineludible del Estado y de la sociedad recrear las condiciones de igualdad de oportunidades por medio de políticas diferenciales o políticas compensatorias que les garanticen iguales condiciones de acceso a los bienes económicos, sociales y culturales (Velandia y Hernández Jaramillo, 2006). A pesar de estas contradicciones, la población con discapacidad requiere de intervenciones estatales para mitigar sus condiciones vulneradas, de menores ofertas de trabajo, menor nivel de educación, mayores necesidades de cuidados y de condiciones de fragilidad en salud (OMS, 20II).
Propiciar un enfoque que avance sobre el modelo social de la discapacidad implica una consolidación de la perspectiva de derechos en el diseño e implementación de acciones públicas, así como la participación de los principales actores sociales involucrados: movimientos sociales de personas con discapacidad, familias y organismos de la sociedad civil.

Por último, resulta necesario profundizar en el análisis de las implicancias teóricas y prácticas de la incorporación de la perspectiva de derechos en la normativa vigente ya que presenta una alternativa a las acciones asistencialistas y biomédicas. 
Abramovich, V. (2006): "Una aproximación al enfoque de derechos en las estrategias y políticas de desarrollo" Revista CEPAL, 88: $35-50$.

Acuña, C. et al. (2010): "Discapacidad: derechos y políticas públicas” en Acuña, C. y Bulit Goñi, L. (comps.): Politicas sobre discapacidad en la Argentina. El desafío de hacer realidad los derechos, Buenos Aires, Siglo Veintiuno.

Acuña, C. y Bulit Goñi, L. G. (2010): Políticas sobre discapacidad en la Argentina. El desafio de hacer realidad los derechos, Buenos Aires: Siglo Veintiuno.

Anaut, S. y Arza, J. (201 5): “La exclusión social en los hogares con algún miembro con discapacidad durante el período de crisis en España”. Revista Española de Discapacidad, 3 (I): 728 .

Arcidiacono, P. (2OI2): "Políticas sociales y bienestar en Argentina 2002-2009. Entre el trabajo asalariado y los programas sociales”. Revista SAAP, 2 (6): 3I9-33I.

Argentina. Ley 22.43 I de Sistema de protección integral de los discapacitados. Boletín Oficial, 20 de Marzo de I98I, núm. 24632.

Argentina. Ley 24.90I de Sistema de Prestaciones Básicas en Habilitación y Rehabilitación Integral a Favor de las Personas con Discapacidad. Boletín Oficial, 5 de diciembre de I997, núm. 28789.

Barnes, C. (I998): "Las teorías de la discapacidad y los orígenes de la opresión de las personas discapacitadas en la sociedad occidental" en L. Barton (comp.): Discapacidad y Sociedad, Madrid, Ediciones Morata-Fundación Paideia.

Barnes, C. (2009): “Un chiste malo: ¿rehabilitar a las personas con discapacidad en una sociedad que discapacita?", en Brogna, P. (comp.): Visiones y revisiones de la discapacidad, México: Fondo de Cultura Económica.

Barragué, B.(2015): La garantía de ingresos mínimos en el igualitarismo (p)redistributivo.
Tesis doctoral. Universidad Autónoma de Madrid.

Belmartino, S. (2009): "Los procesos de toma de decisiones en salud. Historia y teoría". Política y Gestión, II: 33-59.

Bregain, G. (20I4): L'internationalisation imparfaite d'une modernité nord-atlantique: essai d'histoire croisée des politiques publiques $d u$ handicap en Argentine, au Brésil et en Espagne (1956-1982) (en línea). <https://tel.archives-ouvertes.fr/tel-OI037929>, acceso I 4 de diciembre de 2017.

Colectivo IOÉ (1998): Discapacidad y Trabajo en España. Estudio de los procesos de inclusión y exclusión de las personas con discapacidad. Madrid: Ministerio de Trabajo y Asuntos Sociales.

Danani, C. (20I6): "Las políticas públicas del área de desarrollo social durante los gobiernos de Néstor Kirchner y Cristina Fernández”. Análisis, I 2.

Díaz Velázquez, E. (20I6): El acceso a la condición de ciudadanía de las personas con discapacidad: el caso de España. Tesis doctoral. Universidad Complutense de Madrid (en línea). <http://eprints.ucm.es/3783 I/I/T37259.pdf>, acceso I 4 de diciembre de 2017.

Esping-Andersen, G. (2000): Fundamentos sociales de las economías postindustriales, Barcelona: Ariel.

Fantova, F. (20I4): "Las políticas sociales y las familias". Razón y Fe, I392 (270): 32 I-332.

Fara, L. (20I0): “Análisis de la normativa nacional orientada a persona con discapacidad", en Acuña, C. y Bulit Goñi, L. (comps.): Políticas sobre discapacidad en la Argentina. El desafio de hacer realidad los derechos, Buenos Aires: Siglo Veintiuno.

Ferrante, C. (20I4): Renguear el estigma: cuerpo, deporte y discapacidad motriz (Buenos Aires, I950-2010), Buenos Aires: Biblos. 
Fiamberti, H. (2008): "Reflexiones sobre las Políticas Públicas y la legislación vigente. ¿Una oportunidad de cambios?”, en Eroles, C. y H. Fiamberti (comps.): Los derechos de las personas con discapacidad. Análisis de las convenciones internacionales y de la legislación vigente que los garantizan, Buenos Aires: EUDEBA.

Findling, L. (2OI2): "Escenarios de la desregulación de las Obras Sociales Sindicales: el desafío de los actores frente a la libre elección de los servicios de salud. Buenos Aires I997-2004”. Tesis doctoral. Universidad de Buenos Aires.

Findling, L. y López, E. (20 I 5): De cuidados y cuidadoras: acciones públicas y privadas. Buenos Aires: Biblos.

FLACSO (20I4): "Identificación de Barreras de Acceso", en: Barreras de acceso a la salud en Argentina. Mimeo.

Foco/Ceipsu (20I3): "Discapacidad: condiciones de vida y acceso a derechos en la Ciudad de Buenos Aires". Mimeo.

Garcilazo, H. (2006): “La legislación”, en La Discapacidad en Argentina. Un diagnóstico de situación y políticas públicas vigentes al 2005 , Buenos Aires: Fundación Par.

Grassi, E., et al. (1994): Políticas sociales, crisis $y$ ajuste estructural: un análisis del sistema educativo, de obras sociales y de las políticas alimentarias, Buenos Aires: Espacio Editorial.

INDEC (2014): Censo Nacional de Población, Hogares y Viviendas 2010. Censo del Bicentenario. Serie C. Población con dificultad o limitación permanente, Buenos Aires: Instituto Nacional de Estadística y Censos.

Isuani, E. (2009): "El estado de bienestar argentino: un rígido bien durable". Politikós: estudios políticos e internacionales, I2: 35-72.

Jelin, E. (I994): “Familia, Crisis y Después...”, en Wainerman, C. [comp.]. Vivir en Familia. Buenos Aires: Losada.

Joly, E. y Venturiello, M. P. (2OI2): "Persons with Disabilities: Entitled to Beg, Not to Work. The Argentine Case”. Critical Sociology, July I 2: I-23.
Kipen, E. y Vallejos, I. (2009): "La producción de discapacidad en clave de ideología”, en Rosato, A. y Angelino, M. A. (comps.): Discapacidad e ideología de la normalidad, Buenos Aires: Noveduc.

Oliver, M. (I998): “¿Una sociología de la discapacidad o una sociología discapacitada?” en L. Barton (comp.): Discapacidad y Sociedad, Madrid: Ediciones Morata-Fundación Paideia.

OMS y Banco Mundial (20II): Informe mundial sobre la discapacidad (en línea). <http://www. who.int/disabilities/world_report/20I I/es/>, acceso I 4 de diciembre de 2017.

Organización de las Naciones Unidas, ONU (2006). Convención Internacional sobre los Derechos de las Personas con Discapacidad, Nueva York: ONU.

Oszlak, O. y O’Donnel, G. (I98 I): Estado y Políticas Estatales en América Latina: hacia una estrategia de investigación, Buenos Aires: Centro de Estudios de Estado y Sociedad (CEDES).

Pantano, L. (1993): La discapacidad como problema social, Buenos Aires: Eudeba.

Ramacciotti, K. (20I0): "Reflexiones en torno a cómo pensar las intervenciones sociales del Estado". Revista de Estudios Marítimos y Sociales, 3: 193-204.

REDI et al. (2OI2): Informe alternativo: situación de la discapacidad en Argentina 2008/20I 2 (en línea). < http://www.redi.org.ar/Prensa/ Comunicados/Informe-alternativo-alcomite-sobre-los-derechos-de-las-personascon-discapacidad-ONU.pdf $>$, acceso I 4 de diciembre de 2017.

Rodríguez Enríquez, C. (2005): La economía del cuidado: un aporte conceptual para el estudio de políticas públicas, Buenos Aires: CIEPP.

Rosales, P. (2008): “Un estudio general de la Convención Internacional sobre los Derechos de las Personas con Discapacidad". Jurisprudencia Argentina, número especial, III.

Seda, J. (2015): Discapacidad y Derechos: evolución en la legislación y jurisprudencia en la República Argentina. Impacto de la 
Convención Internacional por los Derechos de las Personas con Discapacidad. Tesis doctoral. Universidad de Buenos Aires.

Servicio Nacional de Rehabilitación (2016): Anuario Estadístico de Discapacidad 2015 (en línea). <https://www.snr.gob.ar/wp-content/ uploads/20I 6/og/Anuario-final-20I 5.pdf>, acceso I4 de diciembre de 2017.

SIIS Centro de Documentación y Estudios (20I4): La situación de las personas con discapacidad en el mercado laboral. Informe ejecutivo. Madrid: Fundación Once.

Testa, D. (20II): La lucha contra la poliomielitis. Intermitencias de un recuerdo eludido. Buenos Aires (I943-I97I). Tesis de Maestría en Diseño y Gestión de Políticas y Programas Sociales. FLACSO.

Vallejos, I. (2009): “La categoría de normalidad: una mirada sobre viejas y nuevas formas de disciplinamiento social”, en Rosato, A. y Angelino, M. A. (coords.): Discapacidad e ideología de la normalidad, Buenos Aires: Noveduc.

Velandia, I. C. y Hernández Jaramillo, J. (2006): Exclusión social y discapacidad, Bogotá: Universidad del Rosario.

Venturiello, M. P. (2016): La trama social de la discapacidad: cuerpo, redes familiares y vida cotidiana. Buenos Aires: Biblos. 